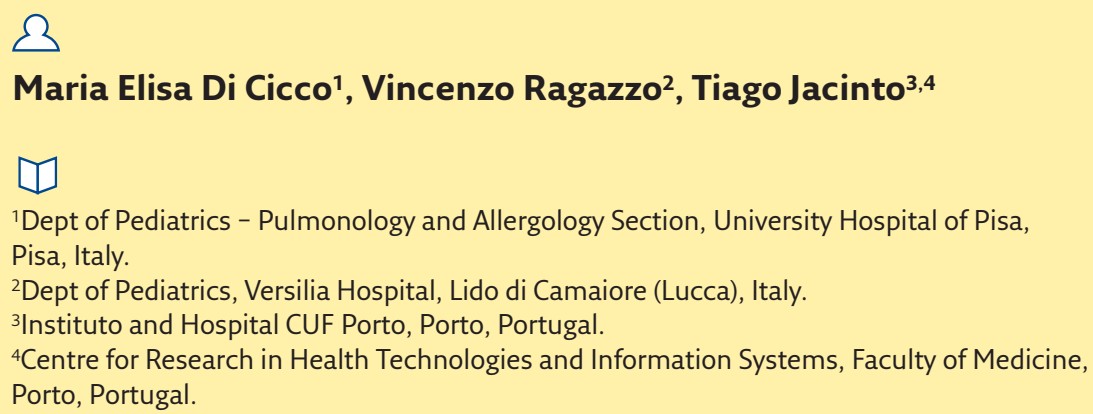

D

'Dept of Pediatrics - Pulmonology and Allergology Section, University Hospital of Pisa,

Pisa, Italy.

2Dept of Pediatrics, Versilia Hospital, Lido di Camaiore (Lucca), Italy.

IInstituto and Hospital CUF Porto, Porto, Portugal.

${ }^{4}$ Centre for Research in Health Technologies and Information Systems, Faculty of Medicine,

Porto, Portugal.

\title{
Mortality in relation to smoking: the British Doctors Study
}

\section{Landmark papers in respiratory medicine}

Today, the uncountable negative effects of cigarette smoking are widely accepted, and it might seem odd to think about the fact that they were largely unsuspected until the 1950s, when many researchers started to study them in different parts of the world.

The work by Richard Doll (English physician, 1912-2005) and Richard Peto (English epidemiologist and statistician, b. 1943), known as the British Doctors Study, is a milestone in this field, since it can be considered the first strong statistical proof of the correlation between smoking habit and many serious diseases, including lung cancer. Remarkably, the study was carried out on a population of doctors, followed up for 50 years.

Everything began in 1950, when two large case-control studies demonstrated that smoking was "a cause, and an important cause" of lung cancer $[1,2]$. One of these studies took place in the UK [1], where in the first half of the 20th century a huge increase in the number of deaths from lung cancer had been observed. The study was conducted by Richard Doll and Austin Bradford Hill (English epidemiologist and statistician, 1897-1991) who demonstrated that the risk of lung cancer was related to the number of cigarettes smoked per day (the risk was 25 times higher in those who smoked $>25$ cigarettes a day than in nonsmokers). At that point, a prospective study to prove the relationship between smoking habit and lung cancer beyond any reasonable doubt was needed. Which is why in 1951, Doll and Hill sent a questionnaire about smoking habits to all the doctors resident in the UK through the British Medical Register. 59600 men and women answered, but data from subjects younger than 35 years old and from females were excluded since lung cancer was rare in these cohorts and to number of women were too few. As a consequence, they collected 34440 questionnaires from male doctors (10118 born before 1900, 7477 born in 1900-1909, 9459 born in 1910-1919, and 7385 born in 1920-1930) and started long-term observation of their mortality. The choice of this cohort was brilliant: as follow-up of UK doctors is easy, since they have to update their names on the British Medical Register to continue to work. Moreover, the collection of data on the causes of death could have been easier in this group of subjects with routine access to high-level medical care.

Doll and Hill could never have imagined that their work would continue for 50 years, becoming the paradigm for cohort studies. Indeed, follow-up of the cohort continued until 2001, and through the decades new questionnaires were sent to
Cite as: Di Cicco ME, Ragazzo V, Jacinto T. Mortality in relation to smoking: the British

Doctors Study. Breathe 2016; 12: $275-276$ 
the study subjects (in 1957, 1966, 1971, 1978, 1991 and 2001) in order to gather information on changes in smoking habits and medical history.

And what did the study find out? After the first 10 years of follow-up [3, 4], Doll and Hill reported 4597 deaths, and described an association between smoking and lung cancer, cancers of the upper respiratory and digestive tracts, chronic bronchitis, pulmonary tuberculosis, coronary disease without hypertension and peptic ulcer. The risk of death from lung cancer was related to the amount of tobacco smoked (the annual death rate was 0.07 per 1000 in nonsmokers and 3.15 per 1000 in men smoking 35 or more cigarettes per day). A reduction of the death rate from lung cancer was seen in those who stopped smoking. Finally, considering the excess deaths in those under the age of 65 years from lung cancer, chronic bronchitis and coronary thrombosis without hypertension as related to cigarette smoking, then mortality from all causes at age 45-64 years was consequently increased by $\sim 50 \%$.

Some years later, Richard Peto joined the research group and published with Doll a number papers on the long-term effects of smoking on these subjects, and in particular 20, 40 and 50 years after the beginning of the study. The famous paper, published in 1976, on the 20-year follow-up clearly showed that smoking was an important cancer risk factor and a cofactor for many other diseases [5]. From 1951 to 1971,10072 deaths were recorded and analysed in detail, demonstrating that between a half and a third of all cigarette smokers were going to die because of their smoking habit (death rate ratio among cigarette smokers and lifelong nonsmokers of comparable age was about 2:1 under 70 years and about $1.5: 1$ over 70 years). Cigarette smoke was associated with 24 causes of death and caused death mainly by heart disease, lung cancer, chronic obstructive lung disease and various vascular diseases. Finally, since many doctors had reduced the amount they smoked during the observation period, a reduction in the incidence of lung cancer was seen, while there was no reduction in the other cancers, further demonstrating the causal association between smoking and lung cancer.

The paper on the 40-year follow-up, published in 1991 [6], showed that excess mortality associated with smoking was about twice as extreme during the second half of the study as it had been during the first half and the authors suggested that half of all regular cigarette smokers would eventually be killed by their habit. Comparing continuing cigarette smokers with lifelong nonsmokers, the death rate ratios were approximately threefold at ages 45-64 years and twofold at ages 65-84 years. Nevertheless, those who stopped smoking before 35 years of age had the same life expectancy as nonsmokers, and those who stopped smoking in middle age could also significantly reduce their risk.

Last but not least, the paper on the 50-year follow-up, published in 2004 [7], confirmed the association between smoking habits and many severe diseases (vascular, neoplastic and respiratory diseases) and the suspected relationship between smoking and 12 types of cancer. In this last part of the study, the authors also found that men born in 1900-1930 who smoked cigarettes continuously died about 10 years younger than lifelong nonsmokers, while stopping at age 60,50 , 40 or 30 years gained about $3,6,9$ or 10 years of life expectancy, respectively.

The British Doctors Study was innovative and remarkable, particularly for the length of the follow-up. Doll and Peto have been rewarded for their commitment to this challenge, as their work, producing a demonstration of the association between cigarette smoking and lung cancer and other severe diseases, contributed to starting the fight against smoking.

\section{Conflict of interest}

None declared.

\section{References}

1. Doll R, Hill AB. Smoking and carcinoma of the lung; preliminary report. Br Med J 1950; 2: 739-748.

2. Wynder EL, Graham EA. Tobacco smoking as a possible etiologic factor in bronchogenic carcinoma; a study of 684 proved cases. J Am Med Assoc 1950; 143: 329-336.

3. Doll R, Hill AB. The mortality of doctors in relation to their smoking habits; a preliminary report. BrMed J 1954; 1: 1451-1455

4. Doll R, Hill AB. Mortality in relation to smoking: ten years' observations of British doctors. BrMed J 1964; 1: 1460-1467.
5. Doll R, Peto R. Mortality in relation to smoking: 20 years' observations on male British doctors. Br Med J 1976; 2 : 1525-1536.

6. Doll R, Peto R, Wheatley $\mathrm{K}$, et al. Mortality in relation to smoking: 40 years' observations on male British doctors. BMJ 1994; 309: 901-911.

7. Doll R, Peto R, Boreham J, et al. Mortality in relation to smoking: 50 years' observations on male British doctors. BMJ 2004; 328: 1519. 